\title{
Epidemiological investigation of Mycoplasma Synoviae in native chicken breeds in China
}

\author{
Shi-Kai Sun ${ }^{1,2}$, Xin Lin², Feng Chen ${ }^{3}$, Ding-Ai Wang ${ }^{2}$, Jun-Peng Lu², Jian-Ping Qin ${ }^{2 *}$ and Ting-Rong Luo ${ }^{1 *}$
}

\begin{abstract}
Backgroud: Mycoplasma synoviae (M. synoviae) is widely distributed around the world, and leads to serious economic losses in the world every year. Nevertheless, the incidence and epidemiology of M. synoviae infection in China have remained unclear.

Results: In this study we demonstrate that over 9773 broiler chicken flocks in 16 Chinese provinces were affected by M. synoviae between 2010 and 2015. Our epidemiological study revealed that M. synoviae was widely prevalent in multi-aged Chinese native breeder chickens, and the prevalence of $M$. synoviae in embryos of breeders reached up to $16.29 \%$. In addition, our data showed that chickens aged 14 days or younger carried simultaneously high levels of maternal antibody against M. synoviae and high M. synoviae infection (10\%), and low M. synoviae antibody levels in breeders and high proportion of M. synoviae infection in embryos could increase the chances of incidence in the offspring. Finally, our results also indicated that 3- to 7-week-old chickens might be most the susceptible to M. synoviae and, therefore, might play a key role in the horizontal transmission of M. synoviae.
\end{abstract}

Conclusion: Our findings suggest that M. synoviae is widely circulating in Chinese native chickens, accordingly, effective control measures are urgently needed to control the spread.

Keywords: Mycoplasma synoviae, Outbreak, Chinese native chicken breeds, Epidemiology

\section{Backgroud}

Mycoplasma synoviae (M. synoviae) is an important disease, causing enormous economic losses to the poultry industry worldwide. M. synoviae infection in chickens or turkeys results in acute or chronic infectious synovitis or air sacculitis [1].

M. synoviae was reported in Australia, South America, Asia, Europe and Africa [2], nevertheless, M. synoviae distribution appeared regional in most cases, and largescale outbreaks rarely occurred. In the past ten years, it seems that $M$. synoviae had taken over the role of Mycoplasma gallisepticum in commercial poultry [3]. Apart from air sacculitis and synovitis, eggshell apex abnormalities (EAA) and egg drop syndrome resulting from M. synoviae infection have been encountered worldwide [3-6]. Hence, there has been a growing emphasis on understanding the prevalence of $M$. synoviae, especially its subclinical infection [3, 7]. Many reports [8-11] world with a varying degree of incidence in multi-age whitefeather chickens or turkeys. To date, there has been no data about $M$. synoviae infection in Chinese native chicken breeds, and the incidence and epidemiology of $M$. synoviae in China are poorly understood.

Between 2010 and 2015, a disease, characteristic of infectious synovitis occurred in native-type chickens in China. The disease resulted in the loss of millions of chickens in Chinese poultry farms. In this study, we conducted systematic epidemiological analysis of this disease.

\footnotetext{
*Correspondence: jpqin@163.com; Tingrongluo@gxu.edu.cn

${ }^{2}$ Guangdong Enterprise Key Laboratory for Animal Health and Environmental

Control, WENS Group Academy, Yunfu, Guangdong 527439, China

${ }^{1}$ State Key Laboratory for Conservation and Utilization of Subtropical

Agro-Bioresources, Guangxi University, Nanning, Guangxi 530004, China

Full list of author information is available at the end of the article

\section{Methods \\ Sample preparation \\ Identification of pathogen}

To determine the causative agent of disease, a total of 18,063 clinical samples (joint fluid swabs and three 
swabs were collected from three sick chickens at each farm) were collected from infected chickens in the farms (Table 1) of 16 Chinese provinces between 2010 and 2015. In addition, from 2010 to 2013, 1696 serum samples were collected from 53 infected broiler flocks (Table 2). Thirty-two blood samples were collected from each farm, including 16 samples from sick chickens with typical clinical signs and 16 samples from chickens without any clinical symptoms.

\section{Prevalence in multi-aged breeder farms}

According to the protocol for an epidemic outbreak, a total of 5760 serum samples were collected from 180 breeder flocks in 6 large-scale multi-age native chicken farms in Guangdong, Guangxi, Hunan and Zhejiang, where the disease occurred in 2013, and detected by ELISA. Chickens ranged from 1 to 40 weeks old, 3-4 flocks were examined for each age (week), totaling 180 flocks with 32 blood samples from each flock. In addition, a total of 1332 7- to 9-day-old embryos (Table 3) were collected from farms in Guangxi, Guangdong, Zhejiang and Hunan in 2013. Allantoic fluid was collected from each embryo for detection of M. synoviae infection.

\section{Natural infection in the offspring of native breeders}

To determine the natural prevalence of $M$. synoviae in broilers, three batches of 1-day-old Three-Yellow chickens (according to the high incidence of $M$. synoviae infection in Three-Yellow broiler chickens) were purchased from a farm in 2014, with 100 chickens in each batch and a 30-day time interval between each batch. Throat swab samples and serum samples of all chickens were collected at $1,5,10,20,30,40,50,60,70,80,90$ and 100 days old, respectively.

\section{Prevalence in different native breeders}

To further understand M. synoviae infection in different native chicken breeds from a farm in Zhejiang, serum and associated embryos collected from three different native chicken breeds (Three-Yellow, Silky, and Qingyuan Ma chickens) were examined. A total of 128 serum samples were simultaneously collected from 3 different chicken breeds (a total of 8 breeder

Table 1 Information of samples for PCR detection and strains isolation

\begin{tabular}{|c|c|c|c|c|c|c|c|}
\hline Province & Sampling city & Breed & Age (day) & $\begin{array}{l}\text { Joint fluid } \\
\text { swabs }^{a}\end{array}$ & $\begin{array}{l}\text { Chicken } \\
\text { legs }^{a}\end{array}$ & $\begin{array}{l}\text { No. of } \\
\text { farm }\end{array}$ & Sampling time \\
\hline Guangdong & $\begin{array}{l}\text { Yunfu, YangJiang, Heyuan, } \\
\text { Zhaoqing, Foshan, Qingyuan, } \\
\text { Jiangmen }\end{array}$ & $\begin{array}{l}\text { Three-Yellow, Chaohua, Silky, } \\
\text { Qingyuan Ma, Wenchang, } \\
\text { Tianlushan, Short footed }\end{array}$ & $29-70$ & 5934 & 61 & 1978 & $2010.10-2014.12$ \\
\hline Guangxi & Nanning, Guilin, Hezhou, Yulin & $\begin{array}{l}\text { Three-Yellow, Chaohua, Silky, } \\
\text { Qingyuan Ma, Short footed }\end{array}$ & $31-75$ & 2004 & 24 & 668 & 2010.09-2014.12 \\
\hline Zhejiang & Quzhou, Huzhou, Jiangshan & Ditto & $30-65$ & 1233 & 24 & 411 & 2012.03-2014.12 \\
\hline Jiangsu & $\begin{array}{l}\text { Zhengjiang, Suzhou, Nanjing, } \\
\text { Yancheng, Suqian, Huaian, } \\
\text { Nantong, Lianyungang, }\end{array}$ & Ditto & $28-68$ & 1809 & 14 & 603 & 2012.01-2014.12 \\
\hline Hunan & Hengyang, Changsha & Ditto & $30-65$ & 1407 & 16 & 469 & 2013.01-2014.12 \\
\hline Hubei & Wuhan, Jingzhou & $\begin{array}{l}\text { Three-Yellow, Chaohua, Silky, } \\
\text { Qingyuan Ma, Wenchang, } \\
\text { Tianlushan, Short footed }\end{array}$ & $42-58$ & 468 & 6 & 156 & 2013.02-2014.12 \\
\hline Anhui & Hefei, Wuhu, Haozhou, Chuzhou & Ditto & $33-71$ & 900 & 4 & 300 & 2013.01-2014.12 \\
\hline Jiangxi & Nanchang, Jian & Ditto & $30-60$ & 1110 & 8 & 370 & 2013.04-2014.12 \\
\hline Sichuang & Meishan, Deyang, Chengdu & Ditto & $32-66$ & 921 & 12 & 307 & $2011.06-2014.12$ \\
\hline Yunnan & Kunming, Dali & Ditto & $26-71$ & 504 & 4 & 168 & 2012.04-2014.12 \\
\hline Guizhou & Qingzhen, Guiyang & Ditto & $31-56$ & 156 & 5 & 52 & 2011.08-2014.12 \\
\hline Chongqing & Chongqing & Ditto & $30-64$ & 297 & 4 & 99 & 2012.03-2014.12 \\
\hline Fujian & Putian, Zhangzhou & $\begin{array}{l}\text { Three-Yellow, Chaohua, Silky, } \\
\text { Qingyuan Ma, Short footed }\end{array}$ & $30-75$ & 723 & 21 & 241 & 2013.02-2014.12 \\
\hline Shangdong & Taian & Three-Yellow, Tianlushan, & $30-52$ & 81 & 3 & 27 & 2013.11-2014.12 \\
\hline Hebei & Cangzhou & $\begin{array}{l}\text { Three-Yellow, Silky, } \\
\text { Qingyuan Ma }\end{array}$ & $33-61$ & 384 & 4 & 128 & $2011.06-2014.12$ \\
\hline Hainan & Haikou, Qionghai & Three-Yellow, Wenchang & $45-65$ & 132 & 4 & 44 & 2014.03-2014.12 \\
\hline Total & & & & 18,063 & 215 & 6021 & 2010-2014 \\
\hline
\end{tabular}

a Joint fluid swabs were collected for PCR detection from 2010; ${ }^{\text {b }}$ Chicken legs were collected for strain isolation between 2013 and 2014 
Table 2 M. synoviae antibody of native broilers infected by infectious synovitis in China

\begin{tabular}{|c|c|c|c|c|c|c|c|c|}
\hline Group & Breed & No. of farm ${ }^{a}$ & Age (day) & Clinical signs & Type of sample & $\begin{array}{l}\text { No. of positive } \\
\text { M. synoviae antibody }\end{array}$ & $\begin{array}{l}\text { Positive } \\
\text { proportion }\end{array}$ & Sampling time \\
\hline A1 & Tianlu & 8 & $35-60$ & Arthrocele & Serum & 79/103 & $76.70 \%$ & 2012.01-2014.12 \\
\hline$A 2$ & Tianlu & & $35-60$ & NO & Serum & $88 / 131$ & $67.18 \%$ & 2012.01-2014.12 \\
\hline B1 & San Huang & 17 & $30-75$ & Arthrocele & Serum & 208/299 & $69.57 \%$ & 2010.10-2014.12 \\
\hline B2 & San Huang & & $30-75$ & NO & Serum & $78 / 252$ & $30.95 \%$ & 2010.10-2014.12 \\
\hline $\mathrm{C} 1$ & Silky & 5 & $31-63$ & Arthrocele & Serum & $59 / 62$ & $95.16 \%$ & 2012.06-2014.12 \\
\hline C2 & Silky & & $31-63$ & NO & Serum & $30 / 80$ & $37.5 \%$ & 2012.06-2014.12 \\
\hline D1 & Hua & 9 & $40-71$ & Arthrocele & Serum & $140 / 155$ & $90.32 \%$ & 2012.02-2014.12 \\
\hline D2 & Hua & & $40-71$ & NO & Serum & $81 / 128$ & $63.28 \%$ & 2012.02-2014.12 \\
\hline E1 & Qingyuan Ma & 14 & $29-62$ & Arthrocele & Serum & $197 / 240$ & $82.08 \%$ & 2011.03-2014.12 \\
\hline E2 & Qingyuan Ma & & $29-62$ & NO & Serum & $112 / 238$ & $47.06 \%$ & 2011.03-2014.12 \\
\hline Total & & 53 & & & & $1072 / 1696$ & $63.20 \%$ & 2010.10-2014.12 \\
\hline
\end{tabular}

${ }^{a}$ Thirty-two blood samples were collected from each farm, including 16 samples from sick chickens with typical clinical signs and 16 samples from chickens without any clinical symptoms

flocks) on December 11, 2013. A total of 223 7- to 9day-old embryos corresponding to these breeder flocks were collected on December 20, 2013. Allantoic fluid was collected from each embryo. Subsequently, 12 offspring broiler flocks corresponding to the breeder flocks mentioned above were tracked for the change of M. synoviae antibody. A total of 478 serum samples were collected from offspring broiler flocks at $4,5,6,7$ weeks old, respectively.

\section{Samples detection}

The presence of $M$. synoviae DNA in swab samples and allantoic fluid was determined by PCR. The presence of M. synoviae antibody in serum samples was determined by ELISA.

\section{Isolation and culture of $M$. synoviae}

A total of 215 joint fluid swabs were sterile collected from the leg joints of $M$. synoviae-infected chickens from farms in Zhejiang, Hunan, Jiangsu, Anhui, Sichuan, Yunnan, Guizhou, Chongqing, Hubei, Hebei, Jiangxi, Fujian, Shandong, Hainan, Guangdong and Guangxi provinces were collected in 2013-2014 (Table 1). One swab sample was added to a tube containing $2 \mathrm{~mL}$ Frey mycoplasma medium (BD Biosciences) with $10 \%$ serum and $1 \%$ thallium-acetate [2]. This sample was then transferred into a $37{ }^{\circ} \mathrm{C}$ incubator $\left(5 \% \mathrm{CO}_{2}\right.$ atmosphere conditions) to culture for 20-24 h; the sample supernatant was removed when the liquid sample color changed from red to brown. After the 4th or 5th passage, pure culture of isolates was used as template and identified directly by PCR and sequenced.The 4th or 5th passage live cell titer was determined based on $50 \%$ color change unit $\left(\mathrm{CCU}_{50}\right)$, as described previously $[12,13]$.

\section{DNA extraction, PCR and sequencing}

Total DNA was extracted directly from joint swab samples and allantoic fluid using the RNeasy kit (AxyPrep, Union City, USA). The presence of $M$. synoviae DNA in swab samples and allantoic fluid was determined by PCR.The TaKaRa PrimeScript one-step PCR Kit Ver.2 (TaKaRa, Dalian, China) was used for PCR. All PCR reactions were carried out using a PCR machine (Biometra, Goettingen, Germany). Samples were detected by PCR as described by Ramirez et al. [14]. A partial sequence of the $v$ lhA gene (1284-1325 bp) of positive samples was amplified by PCR for sequencing as described previously [15]. All sequences from independent PCR products were sequenced 3-4 times in both directions by Sangon Biotech (Shanghai) Co., Ltd.

Table 3 Infection proportion of M. synoviae in embryos of 7 breeder farms in 2013

\begin{tabular}{llllll}
\hline Province & County & No. of farm & No. of positive samples & Positive prevalence & Sampling time \\
\hline Guangdong & Xinxing, Gaoming & 2 & $92 / 250$ & $36.80 \%$ & March, November \\
Hunan & Ningxiang & 1 & $4 / 180$ & $2.22 \%$ & March \\
Guangxi & Yongning & 1 & $73 / 360$ & $20.27 \%$ & March \\
Zhejiang & Nanxun, Wuxing & 3 & $48 / 542$ & $8.85 \%$ & March, July, August \\
& Total & & $217 / 1332$ & $16.29 \%$ & March-November \\
\hline
\end{tabular}

${ }^{\mathrm{a}}$ The 7-9 day-old embryos were collected from 7 breeder farms. Allantoic fluid was collected from each embryo for detection of $M$. synoviae infection by PCR 


\section{Sequence analysis}

After sequencing PCR fragments, nucleotide sequence editing was accomplished using DNAStar (Madison, WI). Multiple-sequence alignments were generated with ClustalX 2.0, and nucleotide sequence homologies were obtained using the Clustal W method of DNAStar. All sequencing data were assembled using DNAStar (Madison, WI). The sequence of each different strain was compared to reference isolates in GenBank.

\section{Serological study}

Serum samples were tested for the presence of $M$. synoviae antibodies using the $M$. synoviae Antibody test kit (IDEXX, Liebefeld-Bern, Switzerland) according to the manufacturer's instructions. Based on the optical density $(O D)$ values at $405 \mathrm{~nm}$, sample to positive $(\mathrm{S} / \mathrm{P})$ ratios were calculated, and the averaged $\mathrm{S} / \mathrm{P}$ ratio was used to evaluate the antibody level in each group. Serum samples with $\mathrm{S} / \mathrm{P}$ ratios greater than 0.2 (titers greater than 1076) were considered to contain anti-M. synoviae antibodies, and a ratio of 0.2 or lower (titers less than or equal to 1076) were considered negative, based on the manufacturer's recommendations.

\section{In vivo experiments}

WVU1853 (M. synoviae standard strain, bought from China Veterinary Drug Supervision), 14 randomly chosen M. synoviae isolates and 160 20-day-old SPF chickens (specific pathogen free chickens, Guangdong Dahuanong Co., LTD) were used for the pathogenicity experiments. 160 chickens were divided into 16 groups, including 15 challenged groups (10 birds/group) and one mock/control group (10 birds). All chickens were kept in SPF chicken isolators (glove box) through the whole experiment. After adaption for three days in the isolator, the chickens were inoculated with fresh cultures of 14 selected isolates and WVU1853 by intramuscular injection with a dose of $10^{7} \mathrm{CCU}_{50}$. Ten chickens served as mock controls and were mock-injected with $0.5 \mathrm{~mL}$ culture medium in the same manner. The chickens were monitored daily while clinical symptoms and morbidity were recorded. All chickens were necropsied on day post-inoculation.

\section{Statistical analysis}

The data obtained were analyzed by one-way analysis of variance (ANOVA), and the differences between means were compared by Duncan's multiple range test (DMRT) using SPSS 17 (SPSS Inc., Chicago, IL, USA). M. synoviae antibody titer and $M$. synoviae antigen-carrying rate were analyzed by Student's t-test. $P<0.05$ was considered to be statistically significant.

\section{Results}

Identification of infectious synovitis caused by M. synoviae infection

In 2010, a disease with characteristic infectious synovitis appeared in chickens in the Guangdong and Guangxi provinces of China (Fig. 1a1). Anatomical examination revealed that visible yellowish purulent exudate or a yellow cheese-like substance accumulated in the joint and wing cavities of sick chickens (Figs. 1a, 2, 3, 4). This disease had a mass rate of $10-100 \%$ and a group morbidity rate reaching $1-20 \%$, with an average of $5-8 \%$. This disease was observed in several Chinese native chicken

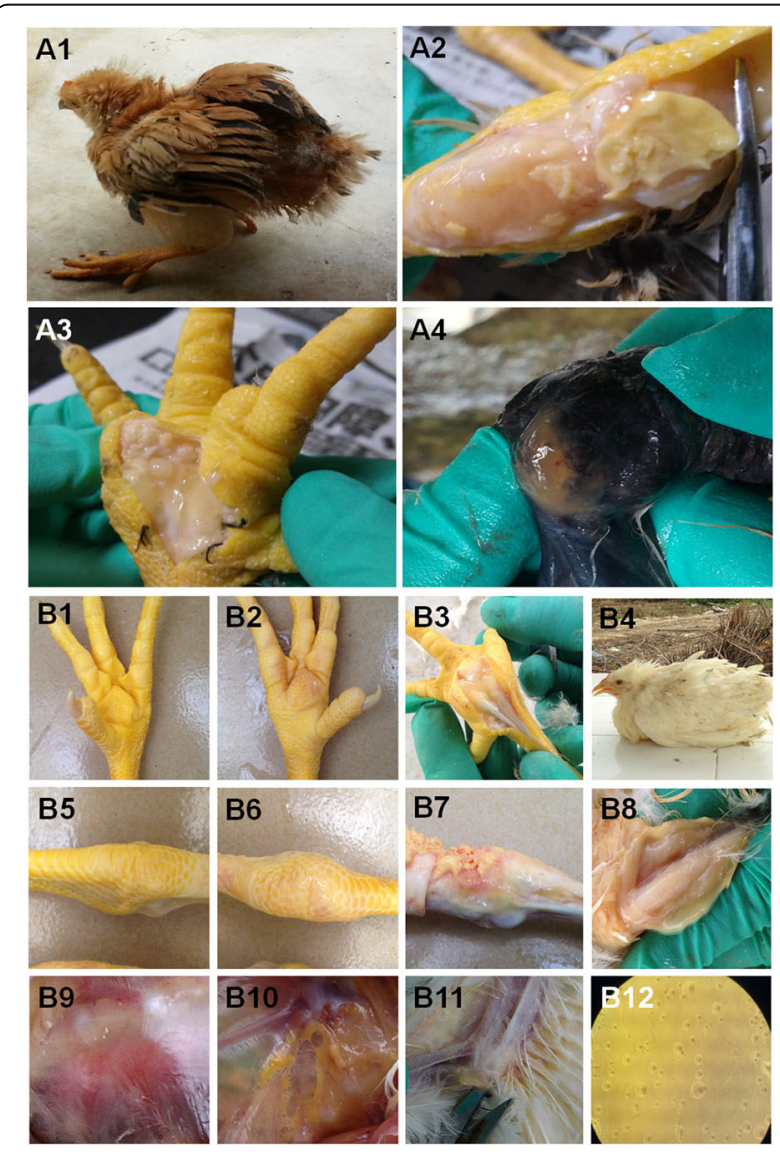

Fig. 1 Pathological studies of infected chickens and inoculated chickens.The infected chickens, which showed clinical symptoms including movement disorders, erect feathers and stunted growth (a1), were killed, and anatomical investigations revealed the presence of thick, clear to milky exudate in enlarged joints (a2, a4) and foot pads (a3). Similar to the clinically infected chickens, the inoculated chickens exhibited symptoms such as movement disorders, erect feathers and stunted growth (b4). The anatomical investigations showed that thick, clear to milky exudate was present in enlarged foot pads (b2, b3), joints (b6, b7), keels (b8) and wings (b11). In addition, air sacculitis (b10) was observed in infected chickens too. Mock-injected chickens' foot pads (b1), joints (b5) and air sacs (b9) were compared with those of the infection chickens. Colonies of M. synoviae were grown in solid medium (b12) 

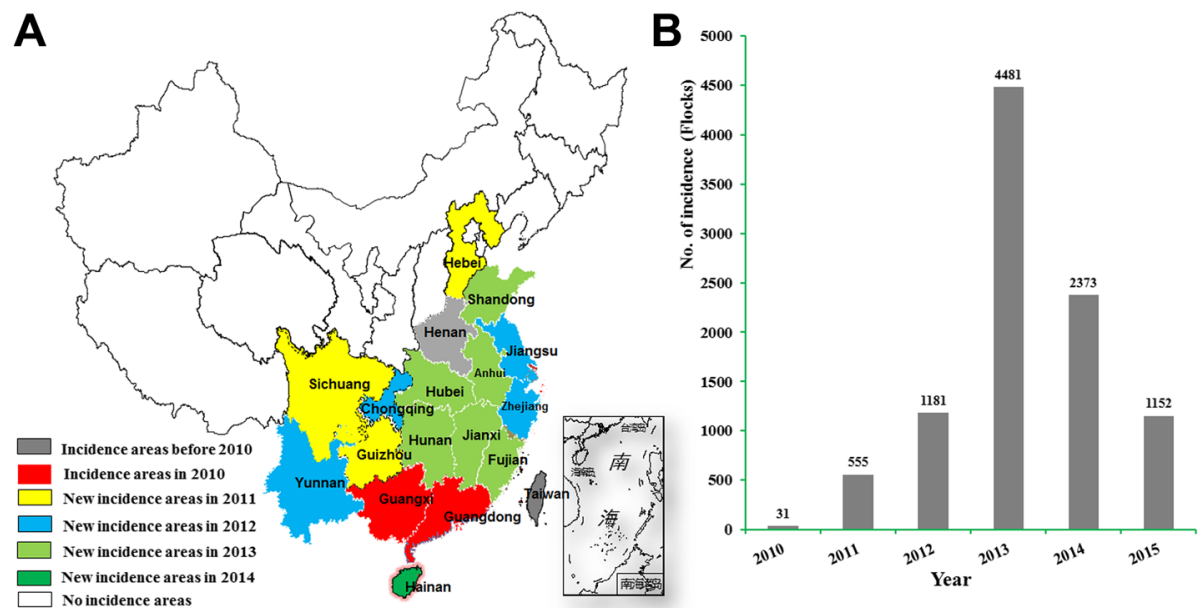

C

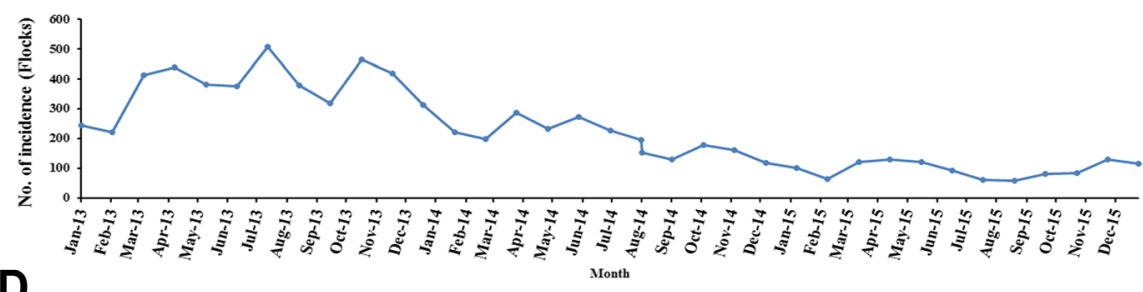

D

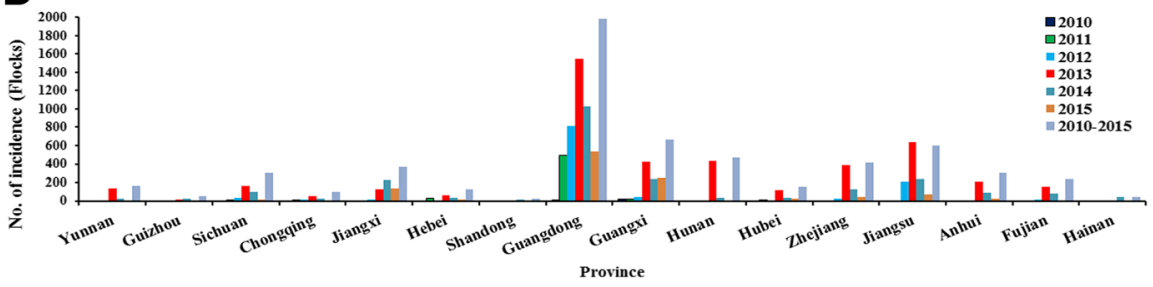

Fig. 2 The prevalence of M. synoviae infection in China between 2010 and 2015.a Incidence in various areas of China from 2010 to 2015 ; bTotal cases of infectious synovitis that appeared in China from 2010 to 2015; c Monthly case occurrence during 2013 and 2015 in China. d Overall cases that appeared in different provinces with reported incidents. (We acknowledge Tang HB for providing the original map of China. The original map was published in the paper by Tang HB et al.: Re-emergence of rabies in the Guangxi province of Southern China. PLoS Negl Trop Dis 2014.8(10):e3114)

breeds, including Three-Yellow, Silky, Qingyuan Ma, Wenchang, Tianlushan, Chaohua and Short-footed chickens.

To determine the causative agent of this disease, we first investigated several pathogens that had previously been reported to associate with infectious synovitis in broiler chickens by pathogenic detection, including Salmonella [16], Reovirus [17, 18], Streptococcus [19, 20], Staphylococcus [21], and M. synoviae [2].The results indicated that no of these pathogens could be detected (data not shown). By contrast, we found that only $M$. synoviae DNA was detected in most of the infected chickens $(17,396 / 18063,96.31 \%)$. Serum antibody analysis also showed the presence of $M$. synoviae antibody in $69.57-95.16 \%$ of the infected chickens, compared to $30.95-67.18 \%$ in clinically healthy chickens of the same flocks (Table 2). The highest prevalence of $M$. synoviae antibody was observed in silky chickens and the lowest in Three-Yellow chickens (Table 2). 110 M. synoviae strains were successfully isolated from $M$. synoviae-infected chickens (Fig. 1b12) and sequenced (Genbank accession number: KU572280-KU572389). Subsequently, typical clinical symptoms were reproduced using part of isolates (Fig. 1b4). Similar to the clinical cases, anatomical examination of experimentally infected chickens revealed that a pale yellow or yellow purulent exudate, cheese-like substance was deposited in the feet, leg joints, wings, and chest (Figs. 1b2, 3, 6, 7, 8, 11), and a proportion of the chickens also exhibited air sacculitis (Fig. 1b10). The experimental M. synoviae strains were successfully recovered from the experimentally infected chickens, and the diagnosis of $M$. synoviae infection was confirmed by $M$. synoviae-specific $\mathrm{PCR}$ and gene sequencing in subsequent experiments (data not shown). 


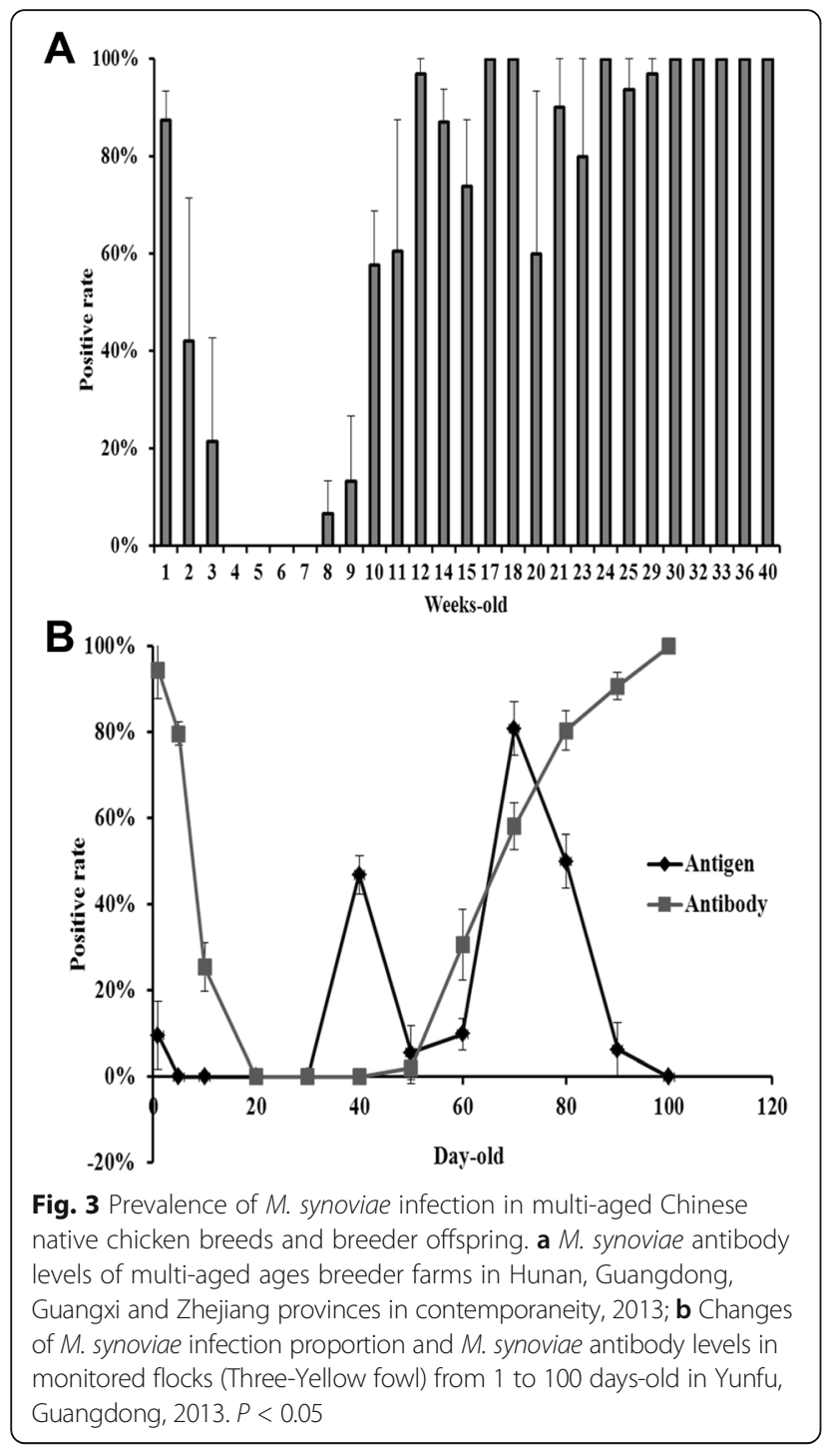

The results revealed that the outbreak of infectious synovitis that emerged in China from 2010 to 2015 was caused by $M$. synoviae infection.

\section{Prevalence of $M$. synoviae infection between 2010 and 2015}

In 2010, the disease initially appeared in the Guangdong and Guangxi provinces of China. The disease spread to larger areas over time, as evidenced by the observation of cases in new provinces since 2011, including Fujian, Zhejiang, Hunan, Jiangsu, Anhui, Sichuan, Yunnan, Guizhou, Chongqing, Hubei, Hebei, Jiangxi, Fujian, Shandong, and Hainan provinces (Fig. 2a). Our epidemiological study showed a dramatic increase in the number of cases from 2010 to 2013 in China, and a slight decrease in 2014 and 2015 (Fig. 2b). The increase may have been associated with a wide range of chicken breeds and a large-scale of cultivation. The disease had

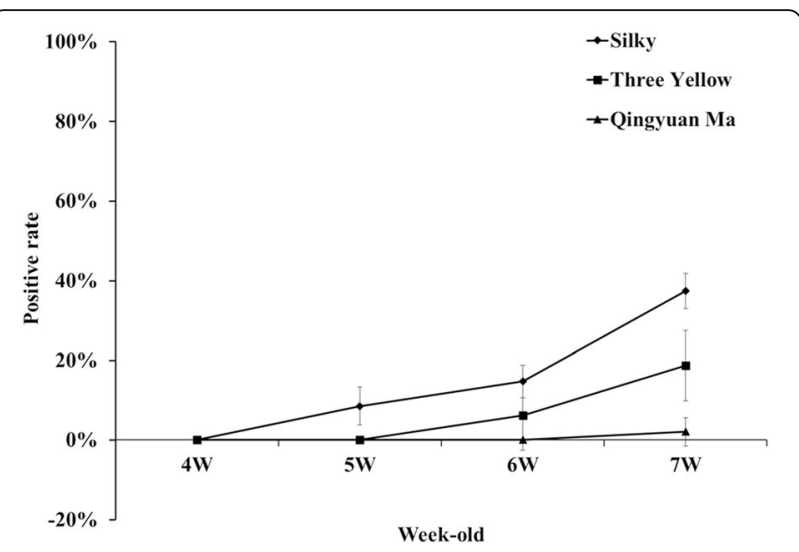

Fig. 4 Changes in M. synoviae antibody levels in several clinical native breed chickens.The 3 breeds of broiler (a total of 12 flocks), including Three-Yellow-, Silky-, and Qingyuan Ma-chicken, were tracking for change of $M$. synoviae antibody at 4, 5, 6, 7 weeks old in the production process, respectively. Positive $M$. synoviae antibody was first observed in the Silky chicken flocks (at 5 weeks-old), but last in the Qingyuan Ma-chicken flocks (at 7 weeks-old). $P<0.05$

no obvious seasonal or breed specificity but did show a slight increase in prevalence at the turn of seasons, i.e. March, April, June, July, September and October (Fig. 2c). The most severe outbreak of the disease occurred in 2013, with the monthly average number of infected flocks reaching approximately 400 (Fig. 2d). It is worth mentioning that Guangdong province, where the incidence was the most serious, had 4417 infected flocks from 2010 to 2015; in 2013 alone, a total of 1547 flocks were infected (Fig. 2d).

Though strong comprehensive measures for prevention and control (including the use of antibiotics and strict implementation of sanitation and disinfection) were carried out after the outbreak of $M$. synoviae, there was still relatively high morbidity in China every year (Fig. 2). Hence, further outbreaks of infectious synovitis have emerged in several native-type chickens in China.

\section{M. synoviae infection prevalence in multi-aged breeder farms}

The results indicated that chickens within a week old normally carried high levels of maternal $M$. synoviae antibody, which declined gradually over the next two weeks. M. synoviae antibody levels in chickens remained negative between 4 to 8 weeks old. The average positive antibody rates of 8-, 9-, 10-, 11-, 12-, 13-, 14-, 15-, 20-, 21-, 23-, 25-, and 29-week-old chickens were 7\%, 13\%, $58 \%, 60 \%, 97 \%$, 87\%, 74\%, 60\%, 90\%, 80\%, 94\%, and 97\%, respectively. The average positive antibody rates of 17-, 18-, 24-, 30-, 32-, 33-, 36-, 40-week-old chickens all reached $100 \%$. M. synoviae antibody levels in $13-$ to $25-$ week-old chickens showed large antibody levels, especially between 20 and 25 weeks old. Multiple variant 
M. synoviae antibody levels were observed among flocks of different ages in this period of time (Fig. 3a).

Subsequently, 1332 embryos from these 6 farms were collected for $M$. synoviae DNA detection. The results showed that the proportion of embryos from Guangdong, Guangxi, Hunan, Zhejiang provinces exhibiting $M$. synoviae infection was $36.8 \%, 20.27 \%$, $2.22 \%$ and $8.85 \%$, respectively (Table 3 ). In addition, the rate of occurrence of eggs with rough head in breeder flocks with lower antibody levels was higher, and the prevalence of $M$. synoviae infection in rough head eggs was significantly higher than that of normal eggs.

These results indicated that Chinese native-breed chickens could carry high levels of $M$. synoviae maternal antibody before the age of 14 days, and there was a widespread $M$. synoviae infection and varying degrees of M. synoviae vertical transmission in multi-age breeder farms.

\section{Natural $M$. synoviae infection in the offspring of native breeders}

The results showed that the change in M. synoviae antibody level in 1- to 20-day-old offspring was consistent with what was observed in the breeder flock described above. The average positive M. synoviae antibody rate, which was over $95 \%$ for 1-day-old chickens, gradually turned negative before the offspring were 20 days old. The chickens remained negative for the $M$. synoviae antibody between 20 and 50 days old. Then, corresponding to the data from breeders, the average positive antibody rate gradually increased from $0 \%$ to $100 \%$ in chickens 50 to 100 days old. These results revealed that $M$. synoviae antibody levels exhibited a gradual, agedependent increase in chicken offspring (Fig. 3b).

At the same time, the results of M. synoviae DNA detection showed that the prevalence of infection in chickens also increased quickly as the offspring aged. There were three time points of peak discharge for excreting bacteria: 1 day old (approximately 10\%), 40 days old (approximately 50\%), and 70 days old (approximately 90\%) (Fig. 3b). Then, the chicken stopped excreting bacteria rapidly. Our study confirmed that chickens can simultaneously carry $M$. synoviae maternal antibody and M. synoviae, and M. synoviae showed a strong capacity for horizontal transmission in Chinese native chicken breeds.

Furthermore, the prevalence of M. synoviae infection in naturally infected chickens showed periodic, agerelated changes. Three- to seven-week-old chickens might be most susceptible to $M$. synoviae infection and, therefore, might play a key role in the horizontal transmission of M. synoviae.

\section{M. synoviae infection of different native chickens on a farm}

The results showed that the majority of flocks exhibited high $M$. synoviae antibody levels, and the average positive rate in seized chickens was $73.33-100 \%$. In 30week-old chickens, the average positive antibody rate could reach $100 \%$, and the average titer could reach 9265 or higher. Flocks of 1005 (Silky chicken) and 1002 (Three-Yellow chicken) had the lowest antibody levels out of all 8 flocks, and the mean titer in these flocks was 2770 and 5648, respectively. Qingyuan Ma chickens had the highest average antibody levels among the three varieties of breeders, while Silky chickens had the lowest (Table 4).

The proportion of M. synoviae infection in embryos of flocks 1001, 1002, 1003, 1004, 1005, 1006, 1007, and 1008 was $4.2 \%, 0.00 \%, 6.67 \%, 7.41 \%, 10.34 \%, 8 \%, 3.45 \%$, and $15.62 \%$, respectively (Table 4 ). The prevalence of $M$. synoviae infection in Three-Yellow chickens, Qingyuan Ma chickens and Silky chickens was 3.78\%, 9.83\%, and

Table 4 Survey of M. synoviae infection in a farm of Zhejiang in December 2013

\begin{tabular}{|c|c|c|c|c|c|c|c|c|c|}
\hline \multirow[t]{2}{*}{ Flock } & \multirow[t]{2}{*}{ Week-old } & \multirow[t]{2}{*}{ Breed } & \multicolumn{4}{|c|}{ Antibody of serum samples from breeder flock ${ }^{b}$} & \multicolumn{3}{|c|}{ Infection proportion of $M$. synoviae in hatching embryos ${ }^{c}$} \\
\hline & & & Positive rate & $\mathrm{AMn}^{\mathrm{a}}$ & CV & No. of sample & No. of positive & Infection proportion & $\begin{array}{l}\text { Infection proportion } \\
\text { of breed }\end{array}$ \\
\hline 1001 & $24 \mathrm{~W}$ & San Huang & $100 \%$ & 5648 & 40 & 16 & $0 / 27$ & $0.00 \%$ & $4.63 \%$ \\
\hline 1002 & $29 \mathrm{~W}$ & San Huang & $100 \%$ & 8634 & 47.6 & 16 & $2 / 27$ & $7.41 \%$ & \\
\hline 1003 & $33 \mathrm{~W}$ & San Huang & $100 \%$ & 9265 & 48.2 & 16 & $1 / 24$ & $4.20 \%$ & \\
\hline 1004 & 39 W & San Huang & $100 \%$ & 10,944 & 40.8 & 16 & $2 / 30$ & $6.67 \%$ & \\
\hline 1005 & $30 \mathrm{~W}$ & Silky & $73.33 \%$ & 2770 & 83.3 & 16 & $3 / 29$ & $10.34 \%$ & $9.25 \%$ \\
\hline 1006 & 54 W & Silky & $100 \%$ & 16,693 & 39.7 & 16 & $2 / 25$ & $8.00 \%$ & \\
\hline 1007 & $35 \mathrm{~W}$ & Qingyuan Ma & $100 \%$ & 11,545 & 34.4 & 16 & $5 / 32$ & $15.62 \%$ & $9.83 \%$ \\
\hline 1008 & $47 W$ & Qingyuan Ma & $100 \%$ & 11,320 & 34.9 & 16 & $1 / 29$ & $3.45 \%$ & \\
\hline
\end{tabular}

${ }^{a}$ Arithmetic mean; ${ }^{b}$ Serum samples were collected from the breeder flocks (1001-1008) on December 11, 2013 and detected by ELISA; ${ }^{7}$-9 days-old embryos corresponding to the breeder flocks (1001-1008) were collected on December 20, 2013. Allantoic fluid was collected from embryos for M. synoviae antigen detection by PCR on December 20, 2013 
$9.25 \%$, respectively (Table 4). The prevalence of $M$. synoviae infection in embryos across all 8 breeder flocks was $7.34 \%$, which was substantially similar to the incidence in broiler chickens (5-8\%), which were the offspring of the 8 breeder flocks in Zhejiang Province.

The progeny broilers originating from these three breeds were tracked for analysis of $M$. synoviae antibody within four weeks (in 4- to 7-week-old chickens). It was found that Silky broiler chickens were positive for the $M$. synoviae antibody earlier than the other two breeds. In Silky broilers were positive for the M. synoviae antibody at five weeks old, while the other two breeds were positive at six weeks old. The prevalence of the antibody in Three-Yellow chickens, Qingyuan Ma chickens and Silky chickens was 5\%,20\% and 40\% at seven weeks old, respectively (Fig. 4).

These results indicated that low $M$. synoviae antibody levels in breeders and high $M$. synoviae antigen positive rate in embryos could increase the chance of incidence in the offspring. Thus, M. synoviae maternal antibody level and $M$. synoviae infection in embryos might affect the incidence of infection in broiler chickens under threat of M. synoviae infection.

\section{Discussion}

In the 1950s, M. synoviae infection was first reported in America [22, 23], after that, researchers came to realize its importance in the poultry industry $[1,2,24,25]$. Prior to this study, there were some single, sporadic reports about white feather chickens affected by $M$. synoviae infection in China [26], but there was a complete lack of data about $M$. synoviae infection in Chinese native chicken breeds. This study is the first to confirm the presence of large epidemics of $M$. synoviae infection in multi-aged farms of native Chinese chicken breeds.

We revealed that chickens could carry the $M$. synoviae maternal antibody and M. synoviae simultaneously before 3 weeks of age, and antibody levels of breeders affected the timing of antibody production and clinical incidence in broiler chickens. The incubation period of $M$. synoviae infection by horizontal transmission was usually 11-21 days but was shorter by vertical transmission (up to only 6 days) [2, 27]. Based on these results, $M$. synoviae antibody and the typical clinical signs of $M$. synoviae infection should be observed in chickens due to vertical transmission of $M$. synoviae at approximately 20 days of age. In fact, although there was vertical transmission, the presence of M. synoviae antibodies in chickens generally could not be detected until 50 days or even later, and the earliest onset of infection in chickens was also not earlier than 35 days in the clinic. Otherwise, although the proportion of embryos with $M$. synoviae infection could exceed $36 \%$, there was no correspondingly high clinical incidence in broiler chickens of Chinese native breeds. It might suggest that, in addition to the potential of chickens to produce anti-M. synoviae antibodies [28] and virulence differences between $M$. synoviae isolates [29-31], maternal antibodies against $M$. synoviae could inhibit $M$. synoviae activity in vivo at early ages and the developmental window when chickens were negative for the M. synoviae antibody - might play a key role in $M$. synoviae reproduction in hosts. Therefore, improving maternal antibody levels in developing chickens and effectively controlling $M$. synoviae activity during the developmental window when chickens are negative for the $M$. synoviae antibody might an efficient strategy to prevent $M$. synoviae infection.

Many immunosuppressive pathogens can interact with $M$. synoviae and lead to serious disease in hosts $[1,32]$. Co-infection of chickens with M. synoviae subclinical infection with avian influenza virus (AIV) H9 [11], infectious bronchitis virus (IBV) [4, 33, 34], Newcastle disease virus (NDV) $[34,35]$ or infectious bursal disease virus (IBDV) [32] caused more severe respiratory or systemic disease than infection with any single abovementioned pathogen. Normally, chickens in China usually suffer from the threat of the above-mentioned situation, but the prevalence of subclinical infection in commercial broilers has not been reported. Consequently, the actual economic loss associated with $M$. synoviae infection might be more serious and larger than it is reported in the press. In this case, we need to attach more importance to $M$. synoviae prevention and control strategies in China.

\section{Conclusions}

In this study, we demonstrated that multi-aged breeder farms are widely infected with $M$. synoviae and that $M$. synoviae is circulating in Chinese native-type chickens. Chinese native-type breeders of 14 days old or younger simultaneously carried high levels of maternal antibodies against $M$. synoviae. In addition, vertical transmission of M. synoviae was observed, and prevalence of $M$. synoviae infection in embryos of breeders reached as high as $16.29 \%$. Finally, our study suggested that 3 - to 7 -weekold chickens might be the most susceptible to M. synoviae infection and may therefore play a key role in the horizontal transmission of M. synoviae.

\section{Abbreviations}

ELISA: Enzyme-linked immunosorbent assay; PCR: Polymerase chain reaction; SPF: Specific pathogen free; $\mathrm{CCU}_{50}$ : $50 \%$ color change unit; SPSS: Statistic package for social science; DNA: Deoxyribose nucleic acid

\section{Acknowledgments}

The authors are thankful to Dr. Yuliang Liu for helpful comments.

\section{Funding}

This work was supported by the Scientific Research Major Project ([WENS] WSSC01-1-C-A-20120525-1419) from Guangdong WENS Foodstuff Group Co, Ltd. 


\section{Availability of data and materials}

The datasets used and/or analysed during the current study available from the first author (E-mail:ssk043@163.com) on reasonable request.

\section{Authors' contribution}

SKS and XL participated in the study design, the experimental work, the analysis interpretation of the data and drafted the manuscript. XL participated in the experimental work, the analysis interpretation of the data and helped draft the manuscript. TRL, JPQ, FC participated in the study design and modify the manuscript. JPL, DAW participated in collecting samples. All authors read and approved the final manuscript.

\section{Competing interests}

The authors declare that they have no competing interests.

\section{Consent for publication}

Not applicable.

\section{Ethics approval and consent to participate}

In this study, all experimental procedures were approved by the Animal Welfare and the Animal Experimental Ethical Committee of Guangxi University (No. Xidakezi2000138). All efforts were made to minimize suffering (Approval No: GXU-AEW-2014-0112). The chicken owners provided consent to collect the samples for privately owned chickens.

\section{Publisher's Note}

Springer Nature remains neutral with regard to jurisdictional claims in published maps and institutional affiliations.

\section{Author details}

'State Key Laboratory for Conservation and Utilization of Subtropical Agro-Bioresources, Guangxi University, Nanning, Guangxi 530004, China. ${ }^{2}$ Guangdong Enterprise Key Laboratory for Animal Health and Environmental Control, WENS Group Academy, Yunfu, Guangdong 527439, China. ${ }^{3}$ College of Animal Science, South China Agricultural University, Guangzhou, Guangdong 510642, China.

Received: 26 October 2016 Accepted: 11 April 2017

Published online: 26 April 2017

\section{Reference}

1. Stipkovits L, Kempf I. Mycoplasmoses in poultry. Revue scientifique et technique (International Office of Epizootics). 1996;15(4):1495-525.

2. Ferguson-Noel SHKaN. Mycoplasma synoviae infection. In: Disease of Poultry (Chinese Version). Edited by Saif.Y.M, 12th edn. Beijing: Blackwell Publishing 2008;999-1014.

3. Landman WJ. Is Mycoplasma synoviae outrunning Mycoplasma gallisepticum? A viewpoint from the Netherlands. Avian Pathol. 2014:43(1):2-8.

4. Feberwee A, de Wit JJ, Landman WJ. Induction of eggshell apex abnormalities by Mycoplasma synoviae: field and experimental studies. Avian Pathol. 2009;38(1):77-85.

5. Catania S, Bilato D, Gobbo F, Granato A, Terregino C, lob L, Nicholas RA. Treatment of eggshell abnormalities and reduced egg production caused by Mycoplasma synoviae infection. Avian Dis. 2010;54(2):961-4.

6. Gole VC, Chousalkar KK, Roberts JR. Prevalence of antibodies to Mycoplasma synoviae in laying hens and possible effects on egg shell quality. Prev Vet Med. 2012;106(1):75-8.

7. Kleven SH. Control of avian mycoplasma infections in commercial poultry. Avian Dis. 2008:52(3):367-74.

8. Hagan JC, Ashton NJ, Bradbury JM, Morgan KL. Evaluation of an egg yolk enzyme-linked immunosorbent assay antibody test and its use to assess the prevalence of Mycoplasma synoviae in UK laying hens. Avian Pathol. 2004; 33(1):93-7.

9. Dufour-Gesbert F, Dheilly A, Marois C, Kempf I. Epidemiological study on Mycoplasma synoviae infection in layers. Vet Microbiol. 2006;114(1-2):148-54.

10. Feberwee A, de Vries TS, Landman WJ. Seroprevalence of Mycoplasma synoviae in Dutch commercial poultry farms. Avian Pathol. 2008;37(6):629-33.

11. Roussan DA, Khawaldeh G, Shaheen IA. A survey of Mycoplasma gallisepticum and Mycoplasma synovaie with avian influenza H9 subtype in meat-type chicken in Jordan between 2011-2015. Poult Sci. 2015;94(7): 1499-503.
12. Branton SL, Leigh SA, Roush WB, Purswell JL, Olanrewaju HA, Collier SD. Effect of selected water temperatures used in Mycoplasma gallisepticum vaccine reconstitution on titer at selected time intervals. Avian Dis. 2008; 52(2):291-6.

13. Stemke GW, Robertson JA. Comparison of two methods for enumeration of mycoplasmas. J Clin Microbiol. 1982;16(5):959-61.

14. Ramirez AS, Naylor CJ, Hammond PP, Bradbury JM. Development and evaluation of a diagnostic PCR for Mycoplasma synoviae using primers located in the intergenic spacer region and the $23 \mathrm{~S}$ rRNA gene. Vet Microbiol. 2006;1 18(1-2):76-82.

15. El-Gazzar MM, Wetzel AN, Raviv Z. The genotyping potential of the Mycoplasma synoviae vlhA gene. Avian Dis. 2012;56(4):711-9.

16. Eyigor A, Goncagul G, Gunaydin E, Carli KT. Salmonella profile in chickens determined by real-time polymerase chain reaction and bacteriology from years 2000 to 2003 in Turkey. Avian Pathol. 2005;34(2):101-5.

17. Liu HJ, Chen JH, Liao MH, Lin MY, Chang GN. Identification of the sigma $\mathrm{C}$-encoded gene of avian reovirus by nested PCR and restriction endonuclease analysis. J Virol Methods. 1999;81 (1-2):83-90.

18. Moradian A, Thorsen J, Julian RJ. Single and combined infections of specificpathogen-free chickens with infectious bursal disease virus and an intestinal isolate of reovirus. Avian Dis. 1990;34(1):63-72.

19. De Herdt P, Desmidt M, Haesebrouck F, Ducatelle R, Devriese LA. Experimental Streptococcus Bovis infections in pigeons. Avian Dis. 1992; 36(4):916-25.

20. Domermuth $\mathrm{CH}$, Gross WB. A medium for isolation and tentative identification of fecal streptococci, and their role as avian pathogens. Avian Dis. 1969;13(2):394-9.

21. Claire BA. Staphylococcosis. In: Disease of Poultry (Chinese Version). Edited by Saif.Y.M, 12th edn. Beijing: Blackwell Publishing. 2008:1059-70.

22. Olson NO, Bletner JK. Enlarged joint condition in poultry caused by an infectious agent. Poult Sci. 1954;33:1075.

23. Anderson GC, Bletner JK, Munro DA, Olson NO, Shelton DC. Studies of infectious synovitis in chickens. Am J Vet Res. 1956;17(65):747-54.

24. Mohammed HO, Carpenter TE, Yamamoto R. Economic impact of Mycoplasma gallisepticum and M. Synoviae in commercial layer flocks. Avian Dis. 1987;31(3):477-82.

25. Olson NO, Ken KM, Campbell A. Control of infectious synovitis. 13. The antigen study of three strains. Avian Dis. 1964;8:209-14.

26. Zhongzan Cao XL. Shengwang Liu, Jianbin he: advance in avian Mycoplasma Synoviae. Prog Vet Med. 2012;11(33):113-7.

27. Senties-Cue G, Shivaprasad HL, Chin RP. Systemic Mycoplasma synoviae infection in broiler chickens. Avian Pathol. 2005;34(2):137-42.

28. Washburn LR, Ramsay JR, Roberts LK. Characterization of the metabolism inhibition antigen of Mycoplasma arthritidis. Infect Immun. 1985;49(2):357-64.

29. Pascucci S, Maestrini N, Govoni S, Prati A. Mycoplasma synoviae in the guinea-fowl. Avian Pathol. 1976;5(4):291-7.

30. Lockaby SB, Hoerr FJ, Lauerman LH, Kleven SH. Pathogenicity of Mycoplasma synoviae in broiler chickens. Vet Pathol. 1998;35(3):178-90

31. Narat M, Bencina D, Kleven SH, Habe F. The hemagglutination-positive phenotype of Mycoplasma synoviae induces experimental infectious synovitis in chickens more frequently than does the hemagglutinationnegative phenotype. Infect Immun. 1998;66(12):6004-9.

32. Giambrone JJ, Eidson CS, Kleven SH. Effect of infectious bursal disease on the response of chickens to Mycoplasma synoviae, Newcastle disease virus, and infectious bronchitis virus. Am J Vet Res. 1977:38(2):251-3.

33. Springer WT, Luskus C, Pourciau SS. Infectious bronchitis and mixed infections of Mycoplasma synoviae and Escherichia coli in gnotobiotic chickens. I. Synergistic role in the airsacculitis syndrome. Infect Immun. 1974:10(3):578-89.

34. Kleven SH, Eidson CS, Anderson DP, Fletcher OJ. Decrease of antibody response to Mycoplasma synoviae in chickens infected with Marek's disease herpesvirus. Am J Vet Res. 1972;33(10):2037-42.

35. Villegas P, Kleven SH, Anderson DP. Effect of route of Newcastle disease vaccination on the incidence of airsacculitis in chickens infected with Mycoplasma synoviae. Avian Dis. 1976;20(2):395-400. 